\title{
ORIGINAL
}

\author{
S. F. Maassen • H. Erdle • S. Pulvermacher • D. Brands $(D$ \\ T. Böhlke · J. Gibmeier • J. Schröder
}

\section{Numerical characterization of residual stresses in a four-point-bending experiment of textured duplex stainless steel}

Received: 21 October 2020 / Accepted: 23 February 2021 / Published online: 19 March 2021

(C) The Author(s) 2021

\begin{abstract}
The resulting shapes in production processes of metal components are strongly influenced by deformation induced residual stresses. Dual-phase steels are commonly used for industrial application of, e.g., forged or deep-drawn structural parts. This is due to their ability to handle high plastic deformations, while retaining desired stiffness for the products. In order to influence the resulting shape as well as component characteristics positively it is important to predict the distribution of phase-specific residual stresses which occur on the microscale of the material. In this contribution a comparative study is presented, where two approaches for the numerical simulation of residual stresses are applied. On the one hand a numerically efficient mean field theory is used to estimate on the grain level the total strain, the plastic strains and the eigenstrains based on macroscopic stress, strain and stiffness data. An alternative ansatz relies on a Taylor approximation for the grain level strains. Both approaches are applied to the corrosion-resistant duplex steel X2CrNiMoN22-5-3 (1.4462), which consists of a ferritic and an austenitic phase with the same volume fraction. Mean field and Taylor approximation strategies are implemented for usage in three dimensional solid finite element analysis and a geometrically exact Euler-Bernoulli beam for the simulation of a four-point-bending test. The predicted residual stresses are compared to experimental data from bending experiments for the phase-specific residual stresses/strains which have been determined by neutron diffraction over the bending height of the specimen.
\end{abstract}

Keywords Residual stresses · Mean field approach $\cdot$ Beam element $\cdot$ Four-point-bending experiment

\section{Introduction}

In microstructured materials, residual stresses are classified based upon their range of influence into three categories. For crystalline materials, macro residual stresses of first kind are homogeneous across large areas of the material, meaning several grains. unzip mat On the microscale of the material, the micro residual stresses

J. Schröder · D. Brands $(\varangle) \cdot$ S. F. Maassen

Institut für Mechanik, Universität Duisburg-Essen, Essen, Germany

E-mail: dominik.brands@uni-due.de

J. Schröder

E-mail: j.schroeder@uni-due.de

T. Böhlke $\cdot$ H. Erdle

Institute of Engineering Mechanics, Karlsruhe Institute of Technology (KIT), Karlsruhe, Germany

E-mail: thomas.boehlke@kit.edu

J. Gibmeier · S. Pulvermacher

Institute of Institute for Applied Materials, Karlsruhe Institute of Technology (KIT), Karlsruhe , Germany

E-mail: jens.gibmeier@kit.edu 
can be sub-divided into residual stresses homogeneous inside a grain, called residual stresses of second kind, and residual stress fluctuations within the grain, called residual stresses of third kind.

The determination of the micro residual stresses is of great interest for the design of materials and the optimization of process parameters. It is however quite challenging to design experiments to gather these data. Experimental residual stress analysis can be classified into destructive (or semi-destructive) and non-destructive methods.

Destructive methods are based on the stress relaxation caused by the cutting or removal of material. Here, the local material removal causes stress redistribution, which can be observed. While destructive methods are attractive for the determination of macro residual stresses, they are not applicable on the micro-scale of the material. Also, destructive methods are not capable to capture residual stresses as they occur in an experiment. They can only provide data at the end of a process. An overview of the characteristics of various residual stress analysis methods is given in [22].

For the non-destructive experimental determination of phase-specific residual stresses, basically diffraction methods can be applied. A well-established diffraction approach for using lab X-radiation bases is the $\sin ^{2} \psi$ method [15]. However, when using soft X-rays (photon energies $<20 \mathrm{keV}$ ) the method is only surface sensitive. For the analysis of residual stress depth distributions the method must be combined with local materials layer removal, e.g., by means of electro-chemical polishing. For non-destructive residual stress analysis in the bulk material high energy synchrotron X-radiation and neutron radiation can be applied. Access to these methods is provided by large scale research facilities. The high penetration depth of neutrons in steel, see [2], allows the analysis of interior residual stresses without local material removal.

It may be concluded that experimental techniques provide only limited insight into the actual stresses and strains within a material. Numerical simulations are often used as a validation layer in the analysis of residual stresses. A common choice in this field is using finite element simulations. To date, there exist various finite element formulations, e.g., volume elements or structural elements. While volume elements are the standard choice, see, e.g., [27], structural elements can be used in cases which allow for certain assumptions on the kinematics, as done in [5], in order to improve performance. Besides the choice of the kinematics and geometry approximation, the material model has a huge influence. When modeling multiphase materials, such as duplex steels, a homogenization technique must be applied. For an overview of various approaches it is referred to Kanouté et al. [10]. In this work, two approaches of differing complexity are compared. A Talor ansatz, which is an effective volume averaging of the phase specific response and a mean field approach (see, e.g., [4]) are considered. Hereby, the mean field approach is implemented as an incremental elasto-plastic constitutive model in $3 \mathrm{~d}$ finite element simulations. Contrary, a classical $J_{2}$ plasticity model is used for the taylor approach together with beam elements as in [5], in a comparatively simplified $2 \mathrm{~d}$ continuum.

The two approaches are applied to the simulation of a four-point-bending test of the two-phase steel X2CrNiMoN22-5-3 (1.4462), which consists of a ferritic and an austenitic phase of equal volume fraction. The characterization of the phase-specific hardening behavior of austenite and ferrite was performed by means of interrupted in-situ tensile tests combined with X-ray diffraction residual stress analysis, according to the $\sin ^{2} \psi$-method, using conventionally generated soft X-rays. Due to the small information depths of soft Xrays for steels this analysis is limited to the near surface region only. Results of these phase-specific analyses were used as input for numerical material modelling. Due to the high amount of both phases and the good separability of the diffraction lines, the material is excellently suited for the analysis of phase-specific residual stresses. However, these tensile data provide limited information as, e.g., only data for isotropic hardening can be extracted.

For validation of the simulated phase-specific stresses a non-destructive, in-situ neutron strain scanning was performed at the instrument SALSA @ ILL, Grenoble (France). This allows the validation of the phase-specific stress distributions over the height of the bending sample at the loaded state as well as after unloading.

In the following, we will introduce the main concepts of the two different material models. In the next chapter, the material characterization is discussed, ending with the concluded sets of material parameters, used for the bending simulation. Then a review of the experimental setup for the four-point bending test is given. Also, some aspects of the specimen preparation and analysis are discussed, as they give a hint on the interpretation of results. Further details on the in-situ measures during the experiment are given. In the subsequent section, the numerical models for the different approaches are presented. The next chapter features an in-depth review of the strains and stresses, during the experiment and numerical analysis. The residual stress distributions are compared. An in depth discussion of the observed results is provided, before ending with some concluding remarks. 


\section{Homogenization schemes and constitutive models}

For the comparative study in this contribution we utilize two models for the homogenization of a duplex steel and apply different established constitutive models to obtain the elasto-plastic stress response. A mean field approach will be used to determine the strain increments and stiffnesses for the individual constituents on the one hand. The second scheme uses a simpler Taylor/Voigt homogenization ansatz, on a total Lagrangian framework.

\subsection{Mean field approach}

In this work a finite element analysis is performed with the commercial software Abaqus 2019 from Dassault Systèmes. Hereby, the mean field approach is implemented as a user material within an objective incremental framework. In order to solve the balance of linear momentum on the macroscale the mean field model is required to give at each integration point the increment of Jaumann stress rate $\Delta \bar{\sigma}$ and the algorithmic tangent based on the deformation gradient $\overline{\boldsymbol{F}}$. The macroscopic strain increment is hereby localized which allows the computation of the phase-specific stress state. In a final step the stress increments within the phases are homogenized to compute the required output of the user material subroutine. For details on the objective stress rate and corresponding tangent modulus it is referred to Hofinger et al. [9]. Note that the Jaumann stress rate, which is used for Abaqus solid continuum elements, leads to an oscillatory response in simple shear deformation, see [14]. The range of deformation where this effect occurs, however, is far beyond the deformation considered in this work.

In the following, time dependencies of quantities are dropped due to better readability. Quantities $\phi$ (of arbitrary tensor rank) describing the phase-specific behavior of a microstructured material, e.g., the Cauchy stress or strain are averaged within each phase $\xi$ with corresponding volume $V_{\xi}$ by $\phi_{\xi}=\langle\phi\rangle_{\xi}=\int_{V_{\xi}} \phi \mathrm{d} V / V_{\xi}$. For a representative volume element (RVE) with volume $V_{\mathrm{RVE}}$ the phase volume fractions are given as $c_{\xi}=$ $V_{\xi} / V_{\mathrm{RVE}}$. The corresponding quantity $\bar{\phi}$ macroscopically effective regarding the RVE results in $\bar{\phi}=\langle\phi\rangle=$ $\sum_{\xi} c_{\xi} \phi_{\xi}$. Willis [26] introduces the localization relations

$$
\boldsymbol{\varepsilon}=\mathbb{A}[\overline{\boldsymbol{\varepsilon}}]-\boldsymbol{a}, \quad \boldsymbol{\sigma}=\mathbb{B}[\overline{\boldsymbol{\sigma}}]-\boldsymbol{b}
$$

which localize the macroscopic strain $\overline{\boldsymbol{\varepsilon}}$ and the macroscopic stress $\overline{\boldsymbol{\sigma}}$ by the strain localization tensor $\mathbb{A}$ and the stress localization tensor $\mathbb{B}$, as well as the corresponding fluctuation fields $\boldsymbol{a}$ and $\boldsymbol{b}$. By using $\overline{\boldsymbol{\varepsilon}}=\langle\boldsymbol{\varepsilon}\rangle$ and $\bar{\sigma}=\langle\sigma\rangle$, the side conditions

$$
\langle\mathbb{A}\rangle=\mathbb{I}^{\mathrm{s}}, \quad\langle\mathbb{B}\rangle=\mathbb{I}^{\mathrm{s}}, \quad\langle\boldsymbol{a}\rangle=\mathbf{0}, \quad\langle\boldsymbol{b}\rangle=\mathbf{0},
$$

are obtained.

The reformulation of a small strain homogenization scheme in an incremental form allows the application in the finite deformation setting. The incremental mean field approach including the algorithmically consistent linearization is presented in detail in Hofinger et al. [9]. In a first step, as proposed by Miehe et al. [17], the explicit incrementally objective formulation of the macroscopic strain increment is constructed as

$$
\Delta \overline{\boldsymbol{\varepsilon}}=\operatorname{Sym}\left(2\left(\overline{\boldsymbol{F}}^{n+1}-\overline{\boldsymbol{F}}^{n}\right)\left(\overline{\boldsymbol{F}}^{n+1}+\overline{\boldsymbol{F}}^{n}\right)^{-1}\right) .
$$

A two-phase material is considered, i.e., $\xi=\{1,2\}$. For the individual phases localization relations similar to Eq. (1) can be constructed

$$
\Delta \boldsymbol{\varepsilon}_{\xi}=\mathbb{A}_{\xi}[\Delta \overline{\boldsymbol{\varepsilon}}]-\Delta \boldsymbol{a}_{\xi}, \quad \Delta \boldsymbol{\sigma}_{\xi}=\mathbb{B}_{\xi}[\Delta \overline{\boldsymbol{\sigma}}]-\Delta \boldsymbol{b}_{\xi},
$$

where $\sigma_{\xi}, \boldsymbol{\varepsilon}_{\xi}$ and $\boldsymbol{\varepsilon}_{\xi}^{\mathrm{p}}$ denote the phase-specific stress, total strain and plastic strain respectively. As shown in Hofinger et al. [9] the phase-specific strain localization tensors $\mathbb{A}_{\xi}$ and phase-specific stress localization tensors $\mathbb{B}_{\xi}$ result from the Hill condition [8] and Hooke law as

$$
\begin{array}{ll}
\mathbb{A}_{1}=\frac{1}{c_{1}}\left(\mathbb{C}_{1}-\mathbb{C}_{2}\right)^{-1}\left(\overline{\mathbb{C}}-\mathbb{C}_{2}\right), & \mathbb{A}_{2}=\frac{1}{c_{2}}\left(\mathbb{C}_{2}-\mathbb{C}_{1}\right)^{-1}\left(\overline{\mathbb{C}}-\mathbb{C}_{1}\right), \\
\mathbb{B}_{1}=\frac{1}{c_{1}}\left(\mathbb{S}_{1}-\mathbb{S}_{2}\right)^{-1}\left(\overline{\mathbb{S}}-\mathbb{S}_{2}\right), & \mathbb{B}_{2}=\frac{1}{c_{2}}\left(\mathbb{S}_{2}-\mathbb{S}_{1}\right)^{-1}\left(\overline{\mathbb{S}}-\mathbb{S}_{1}\right),
\end{array}
$$


where $\overline{\mathbb{C}}$ denotes the stiffness tensor of the effective material. Furthermore, the phase-specific strain fluctuation fields $\Delta \boldsymbol{a}_{\xi}$ and phase-specific stress fluctuation fields $\Delta \boldsymbol{b}_{\xi}$ are given as

$$
\begin{aligned}
\Delta \boldsymbol{a}_{1} & =\frac{1}{c_{1}}\left(\mathbb{C}_{1}-\mathbb{C}_{2}\right)^{-1}\left(\overline{\mathbb{C}}\left[\Delta \overline{\boldsymbol{\varepsilon}}^{\mathrm{p}}\right]-c_{1} \mathbb{C}_{1}\left[\Delta \boldsymbol{\varepsilon}_{1}^{\mathrm{p}}\right]+c_{2} \mathbb{C}_{2}\left[\Delta \boldsymbol{\varepsilon}_{2}^{\mathrm{p}}\right]\right), \\
\Delta \boldsymbol{a}_{2} & =\frac{1}{c_{2}}\left(\mathbb{C}_{2}-\mathbb{C}_{1}\right)^{-1}\left(\overline{\mathbb{C}}\left[\Delta \overline{\boldsymbol{\varepsilon}}^{\mathrm{p}}\right]-c_{1} \mathbb{C}_{1}\left[\Delta \boldsymbol{\varepsilon}_{1}^{\mathrm{p}}\right]+c_{2} \mathbb{C}_{2}\left[\Delta \boldsymbol{\varepsilon}_{2}^{\mathrm{p}}\right]\right), \\
\Delta \boldsymbol{b}_{1} & =\frac{1}{c_{1}}\left(\mathbb{S}_{2}-\mathbb{S}_{1}\right)^{-1}\left(\Delta \overline{\boldsymbol{\varepsilon}}^{\mathrm{p}}-c_{1} \Delta \boldsymbol{\varepsilon}_{1}^{\mathrm{p}}-c_{2} \Delta \boldsymbol{\varepsilon}_{2}^{\mathrm{p}}\right), \\
\Delta \boldsymbol{b}_{2} & =\frac{1}{c_{2}}\left(\mathbb{S}_{1}-\mathbb{S}_{2}\right)^{-1}\left(\Delta \overline{\boldsymbol{\varepsilon}}^{\mathrm{p}}-c_{1} \Delta \boldsymbol{\varepsilon}_{1}^{\mathrm{p}}-c_{2} \Delta \boldsymbol{\varepsilon}_{2}^{\mathrm{p}}\right) .
\end{aligned}
$$

Potential relations for the phase-stresses are obtained by the application of the Coleman-Noll procedure to the Clausius-Duhem inequality, see Coleman and Noll [3]. The assumption of a purely energetic Cauchy stress based on a quadratic form of the elastic free energy function of the phases results in the incremental form of the Hooke law

$$
\Delta \boldsymbol{\sigma}_{\xi}=\mathbb{C}_{\xi}\left[\Delta \boldsymbol{\varepsilon}_{\xi}-\Delta \boldsymbol{\varepsilon}_{\xi}^{\mathrm{p}}\right]
$$

with phase-specific stiffness tensors $\mathbb{C}_{\xi}$. The plastic strain rate of the phases are given by

$$
\dot{\boldsymbol{\varepsilon}}_{\xi}^{\mathrm{p}}=\dot{\varepsilon}_{0 \xi}^{\mathrm{p}}\left(\frac{\sqrt{3 / 2}\left\|\boldsymbol{\sigma}_{\xi}^{\prime}\right\|-\sigma_{\xi}^{\mathrm{F} 1}}{\sigma_{\xi}^{\mathrm{F} 2}}\right)^{m_{\xi}} \frac{\boldsymbol{\sigma}_{\xi}^{\prime}}{\left\|\boldsymbol{\sigma}_{\xi}^{\prime}\right\|}
$$

with the stress exponent $m_{\xi}$ and the reference strain rate $\dot{\varepsilon}_{0 \xi}^{\mathrm{p}}$. For sake of simplicity the flow stress $\sigma_{\xi}^{\mathrm{F} 1}$ is neglected while the flow stress $\sigma_{\xi}^{\text {F2 }}$ is modeled by a phenomenological Voce-type hardening approach based on the equivalent plastic strain $\varepsilon_{\xi}^{\mathrm{eq}}=\int\left\|\dot{\boldsymbol{\varepsilon}}_{\xi}^{\mathrm{p}}\right\| \mathrm{d} t$ as

$$
\sigma_{\xi}^{\mathrm{F} 2}=\sigma_{\xi}^{\mathrm{F} 0}+\left(\sigma_{\xi}^{\mathrm{F} \infty}-\sigma_{\xi}^{\mathrm{F} 0}\right)\left(1-\exp \left(\frac{\Theta_{\xi}^{\infty}-\Theta_{\xi}^{0}}{\sigma_{\xi}^{\mathrm{F} \infty}-\sigma_{\xi}^{\mathrm{F} 0}} \varepsilon_{\xi}^{\mathrm{eq}}\right)\right)+\Theta_{\xi}^{\infty} \varepsilon_{\xi}^{\mathrm{eq}} .
$$

Hereby, the initial yield stress $\sigma_{\xi}^{\mathrm{F} 0}$, the saturation stress $\sigma_{\xi}^{\mathrm{F} \infty}$, the initial hardening modulus $\Theta_{\xi}^{0}$ and the saturation hardening modulus $\Theta_{\xi}^{\infty}$ are introduced as material parameters. Note, that in this work plastic isotropy is considered. A modeling approach for an extension of Eq. (8) to plastic anisotopy is presented in Böhlke et al. [1]. For a simulative and experimental investigation of residual stresses within duplex steel with consideration of a correlation between elastic and plastic anisotropy it is referred to Simon et al. [24].

\subsection{Taylor approach}

To account for the duplex steel character in a simplified manner, a Taylor/Voigt approximation is chosen. The constitutive law is evaluated twice at each integration point, using the material parameters of each constituent, namely Austenite $(\xi=1)$ and Ferrite $(\xi=2)$ individually. Following the Taylor scheme, the resulting homogenized first Piola-Kirchhoff stresses $\overline{\boldsymbol{P}}$ and consistent elasto-plastic tangent moduli $\overline{\mathbb{A}}$ are computed by

$$
\overline{\boldsymbol{P}}=c_{1} \boldsymbol{P}_{1}+c_{2} \boldsymbol{P}_{2} \text { and } \overline{\mathbb{A}}=\partial_{\overline{\boldsymbol{F}}} \overline{\boldsymbol{P}}=c_{1} \partial_{\overline{\boldsymbol{F}}} \boldsymbol{P}_{1}+c_{2} \partial_{\overline{\boldsymbol{F}}} \boldsymbol{P}_{2},
$$

with $c_{1}$ and $c_{2}$ being the volume fractions of the constituents. For the constitutive model a finite strain elastoplastic formulation was chosen. Here we employ the well-known concept of an multiplicative decomposition of the deformation gradient resulting in the elastic deformation represented by

$$
\boldsymbol{F}_{\xi}^{\mathrm{e}}=\overline{\boldsymbol{F}} \boldsymbol{F}_{\xi}^{\mathrm{p}-1} \text { and } \boldsymbol{C}_{\xi}^{\mathrm{e}}=\boldsymbol{F}_{\xi}^{\mathrm{e} \mathrm{T}} \boldsymbol{F}_{\xi}^{e}
$$




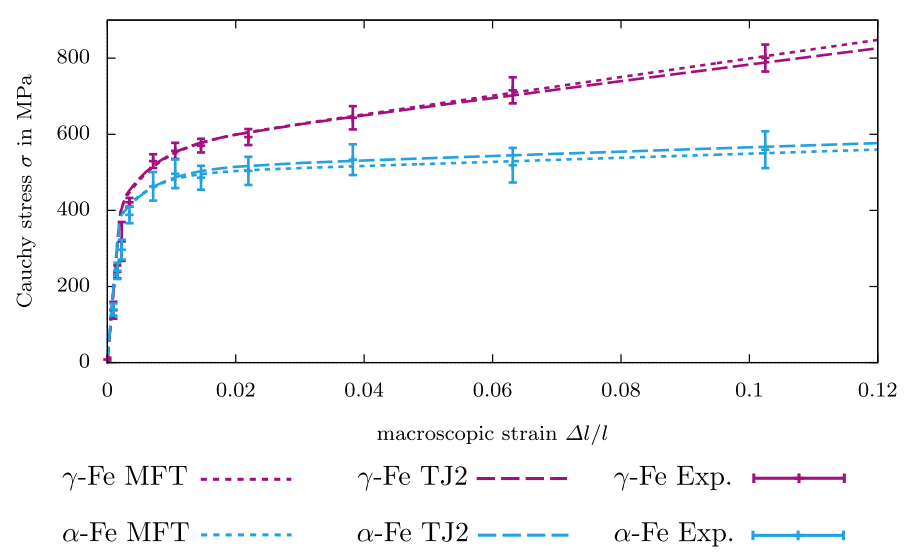

Fig. 1 Uniaxial tensile test simulation results based on mean field theory (MFT) and Taylor approach with the $\mathrm{J}_{2}$ formulation (TJ2). The phase-specific Cauchy stresses for austenite and ferrite are shown versus the macroscopic strain $(\Delta l / l)$. Experimental results of Simon et al. [24]

where $\boldsymbol{F}_{\xi}^{\mathrm{e}}$ and $\boldsymbol{C}_{\xi}^{\mathrm{e}}$ represent the elastic part of the deformation gradient and the elastic right Cauchy-Green deformation tensor, for each of the constituents, respectively. The stresses, based on elastic deformations are obtained from a nonlinear Neo-Hookean free energy

$$
\psi_{\xi}=\frac{\lambda_{\xi}}{4}\left(\operatorname{det}\left(\boldsymbol{C}_{\xi}^{\mathrm{e}}\right)-1-\ln \left(\operatorname{det} \boldsymbol{C}_{\xi}^{\mathrm{e}}\right)\right)+\frac{\mu_{\xi}}{2}\left(\operatorname{tr}\left(\boldsymbol{C}_{\xi}^{\mathrm{e}}\right)-3-\ln \left(\operatorname{det} \boldsymbol{C}_{\xi}^{\mathrm{e}}\right)\right)
$$

with the phase specific Lame constants $\lambda_{\xi}$ and $\mu_{\xi}$. The plastic flow is triggered by a von Mises flow criteria to the Kirchhoff stresses $\tau$

$$
\phi=\sqrt{\frac{2}{3} \operatorname{dev}\left(\boldsymbol{\tau}_{\xi}\right) \operatorname{dev}\left(\boldsymbol{\tau}_{\xi}\right)}-\tau_{y, \xi} \leq 0
$$

where the yield surface evolves by linear and exponential hardening. For a better comparison of the results we employ the same yield stress formulation as introduced in Eq. (9), but formulated in the Kirchhoff stresses. If for any constituent the yield criterion demands plastic evolution, an associative flow rule is employed. Implicit time integration is used to update the phase specific internal variables of plastic flow. In our case we used $\boldsymbol{F}_{\xi}^{\mathrm{p}-1}$ and $\varepsilon_{\xi}^{\mathrm{eq}}$. Implementation and theory of finite plasticity is intensively covered in the literature. As a starting point the interested reader is referred to, e.g., [23].

\section{Identification of material parameters}

The material parameters are obtained based on the experimental results of Simon et al. [24]. In their study the strain hardening of ferrite and austenite are investigated for a cold rolled duplex stainless steel of type $\mathrm{X} 2 \mathrm{CrNiN} 23-4$, which resembles the duplex stainless steel investigated in this work. Phase-specific X-ray diffraction stress analysis were carried out for the $\{220\}$-austenite and the $\{211\}$-ferrite diffraction lines according to the $\sin ^{2} \psi$-method using Vanadium-filtered $\mathrm{CrK} \alpha$-radiation for samples being stepwise loaded in tension up to total strain of about $10 \%$. The uniaxial loading was carried out using a miniature tensile/compression module DDS-2 from Kammrath and Weiss GmbH, Germany. For further experimental details it is referred to [24]. Although the experiments were carried out in three different directions with respect to the rolling direction, due to the assumption of an isotropic plastic material behavior, here only the data from the experiment in transverse direction is considered. The experimental results show that the transverse orientation is least affected by texture-based evaluation errors. The elastic constants were determined based on the orientation distribution function and single-crystal elastic constants, while the initial yield stresses were estimated based on the evolution of integral breadths X-ray diffraction lines. The effective stiffness is approximated using the geometric mean of Lame's constants [16]. This procedure is in accordance with [24].

For the mean field theory (MFT) the phase-specific material parameters of the hardening curve, Eq. (9), are fitted with a least-squares optimization using the Levenberg-Marquardt algorithm. The hardening curves 
Table 1 Material parameter of austenite and ferrite for X2CrNiMoN22-5-3 based on uniaxial tensile tests of Simon et al. [24]. Elastic properties are computed based on the orientation distribution function with single crystal elastic constants of austenite [13] and ferrite [11].

\begin{tabular}{|c|c|c|c|c|c|}
\hline & & Austenite & & Ferrite & \\
\hline & & $\mathrm{TJ} 2$ & MFT & $\mathrm{TJ} 2$ & MFT \\
\hline \multirow[t]{3}{*}{ Single crystal elastic constants } & $C_{11}$ & $233 \mathrm{GPa}$ & & $230 \mathrm{GPa}$ & \\
\hline & $C_{12}$ & $163 \mathrm{GPa}$ & & $135 \mathrm{GPa}$ & \\
\hline & $C_{44}$ & $123 \mathrm{GPa}$ & & $114 \mathrm{GPa}$ & \\
\hline Young's modulus & $E$ & $197 \mathrm{GPa}$ & & $211 \mathrm{GPa}$ & \\
\hline Poisson ratio & $v$ & 0.31 & & 0.29 & \\
\hline Initial yield stress & $\sigma^{\mathrm{F} 0}$ & $420 \mathrm{MPa}$ & & $380 \mathrm{MPa}$ & \\
\hline Saturation stress & $\sigma^{\mathrm{F} \infty}$ & $564 \mathrm{MPa}$ & $573 \mathrm{MPa}$ & $509 \mathrm{MPa}$ & $480 \mathrm{MPa}$ \\
\hline Initial hardening & $\Theta^{F 0}$ & $39.21 \mathrm{GPa}$ & $35.5 \mathrm{GPa}$ & $30.8 \mathrm{GPa}$ & $21.8 \mathrm{GPa}$ \\
\hline Saturation hardening & $\Theta^{\infty}$ & $2.95 \mathrm{GPa}$ & $3.0 \mathrm{GPa}$ & $75.6 \mathrm{MPa}$ & $90 \mathrm{MPa}$ \\
\hline
\end{tabular}

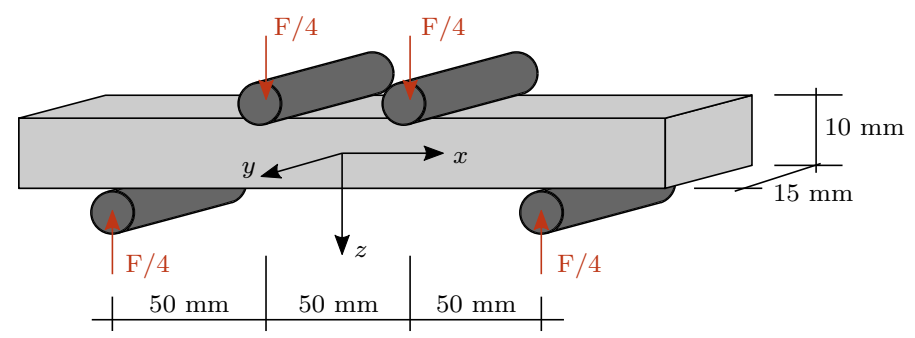

Fig. 2 Four-point-bending setup: sample dimensions and positions of the support pins or the loading pins, respectively

are fitted to experimental data from uniaxial tensile tests. Hereby, the phase-specific stresses are obtained as a function of the macroscopic strain in tensile direction. The material parameter fit, described in detail in Simon et al. [24], is based on the Voigt-type approach, i.e., $\boldsymbol{\varepsilon}_{\xi}=\overline{\boldsymbol{\varepsilon}}$. Even though only one component of the effective stress is nonzero for the uniaxial tensile test, due to the heterogenous material the measured phase-specific stress component in loading direction is insufficient for a fit of the material parameters. To overcome this shortcoming additional assumptions are required. It is assumed that the stress fluctuation field $\boldsymbol{b}$ has the same direction as the effective plastic strain $\overline{\boldsymbol{\varepsilon}}_{\xi}^{\mathrm{p}}$. Furthermore, it is assumed that the directions of the local plastic strains do not change during the uniaxial tensile test. Under this assumption it follows from Eq. (8) that the direction of the local plastic strain $\varepsilon_{\xi}^{\mathrm{p}}$ is equal to the direction of the deviatoric part of the local stress $\boldsymbol{\sigma}_{\xi}^{\prime}$. The material parameters used in the Taylor approach with the $\mathrm{J}_{2}$ formulation (TJ2), see Sect. 2.2, has been obtained by a hand fitted least-squares approach, against the experimental data. These parameter were fitted individually for each phase.

In Table 1 all material parameters for both model approaches are listed. Figure 1 shows the results of uniaxial tensile simulations in comparison with the experimentally obtained results of Simon et al. [24]. The difference of material parameters is obtained as the fitting of the constituent parameters was performed on the homogenized two phase materials. The different homogenization approaches and assumptions, explain the deviations.

\section{Four-point-bending experiment}

\subsection{Experimental setup}

For the experimental residual stress analysis a bending beam of dimensions $160 \mathrm{~mm}$ length, $15 \mathrm{~mm}$ depth and $10 \mathrm{~mm}$ height was cut from a circular rod of X2CrNiMoN22-5-3 with a diameter of $30 \mathrm{~mm}$. The distance of the supporting pins and the loading pins of the experimental setup was $50 \mathrm{~mm}$. Figure 2 shows the sample geometry with indication of the sample dimension and the position of the support pins for four-point bending.

The sectioning of the bending beam was carried out slightly off the rod axis, which causes an heterogeneous distribution of the phase-specific crystallographic texture over the bending height. The texture distribution of the ferrite and the austenite phase over the bending beam height was determined by means of X-ray diffraction 


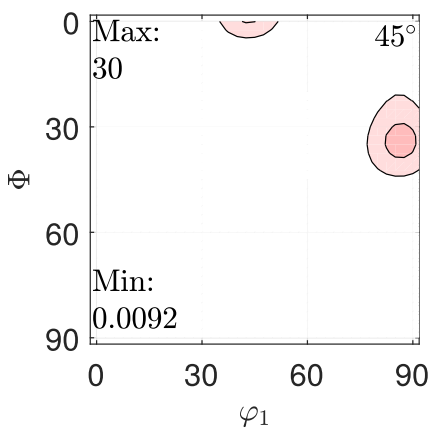

(a) $\alpha$-Fe, $z=-4.9 \mathrm{~mm}$

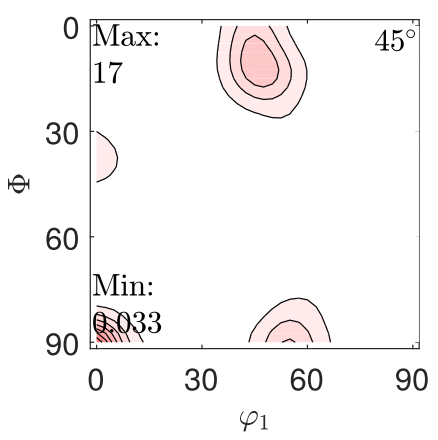

(d) $\gamma$-Fe, $z=-4.9 \mathrm{~mm}$

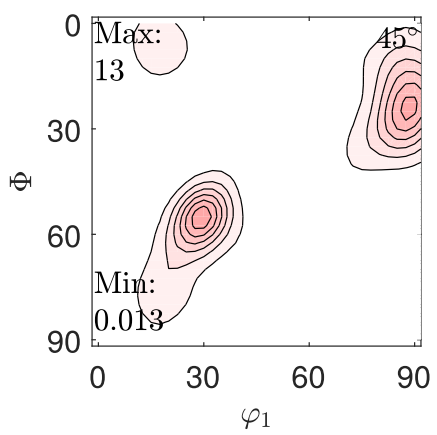

(b) $\alpha$-Fe, $z=+0.8 \mathrm{~mm}$

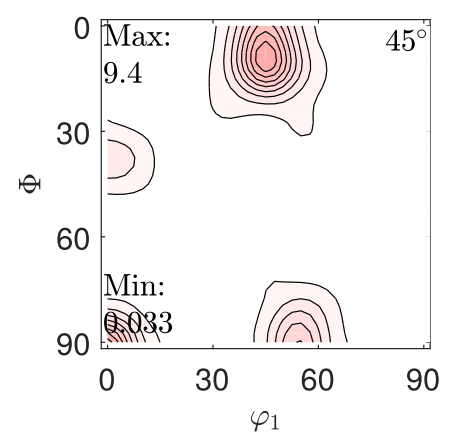

(e) $\gamma$-Fe, $z=-0.8 \mathrm{~mm}$

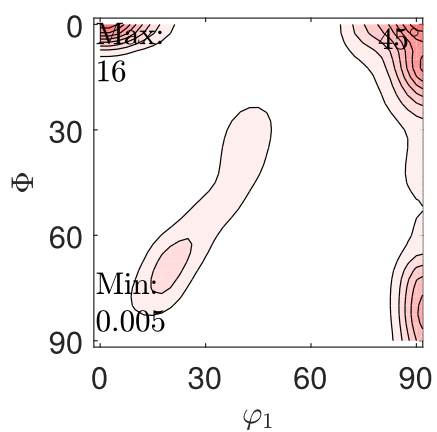

(c) $\alpha-\mathrm{Fe}, z=+4.9 \mathrm{~mm}$

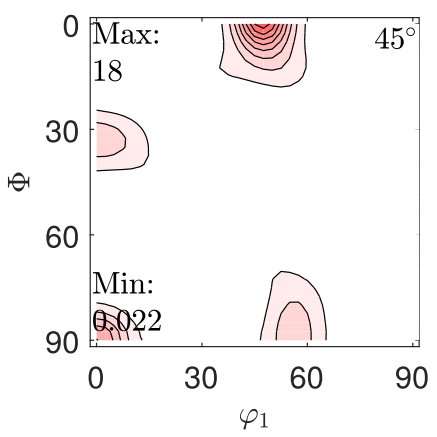

(f) $\gamma-\mathrm{Fe}, z=+4.9 \mathrm{~mm}$

Fig. 3 ODF sections $\Phi 2=45^{\circ}$ for different positions of the bending beam for the ferrite phase (top) and austenite phase (bottom)

for a bending beam that was layer-wise sectioned using 10 layers in total. Phase-specific texture analysis was carried out after electro-chemical polishing of the sectioned layers to prevent an effect of the cutting operation on the diffraction results. Figure 3 shows selected sections of ODFs (Orientation Distribution Function) as an example, determined for austenite and ferrite for different positions of the bending beam. The positions $z=-4.9 \mathrm{~mm}$ and $z=+4.9 \mathrm{~mm}$ correspond to positions near to the outer layers of the surface that were later loaded in compression and in tension, respectively. Furthermore, the mid layer position is considered. Without going into detail to the ODF interpretation the selected graphs clearly indicate that the bending beam exhibit phase-specific texture, which is typical for bcc (ferrite) and fcc (austenite) materials manufactured this way. However, the ODF cuts furthermore indicate that in particular for the ferrite phase significantly varies with position. The texture as well as the gradient in phase-specific crystallographic texture surely affects the phase-specific elasto-plastic behavior.

The neutron strain analyses were performed at the SALSA instrument at the neutron research reactor ILL, Grenoble (France) using a neutron wavelength of 1.6 ̊. Figure 4 presents a detailed view of the applied setup showing the bending device mounted on the Eulerian cradle of the SALSA instrument. In addition, the radial collimators applied on the primary and on the secondary side of the beam paths are shown, respectively, which defined a nominal gauge volume of $0.6 \times 0.6 \times 10 \mathrm{~mm}^{3}$. The cross section $0.6 \times 0.6 \mathrm{~mm}^{2}$ coincides with the cross section of the bending beam, i.e., with the $y-z$ plane. The lattice strain determinations were performed non-destructively for all three principal directions $x, y$ and $z$ on the $\{211\}$ and the $\{220\}$ lattice planes of the ferrite phase as well as on the $\{220\},\{311\}$ and $\{220\}$ lattice planes of the austenite phase in a $2 \Theta$-range between about $75-107^{\circ}$ by shifting the bending beam stepwise through the nominal gauge volume (classical neutron strain scanning experiment). The individual diffraction lines were fitted using a Gaussian function by means of the software package LAMP [20]. For strain calculation the lattice parameters determined for 


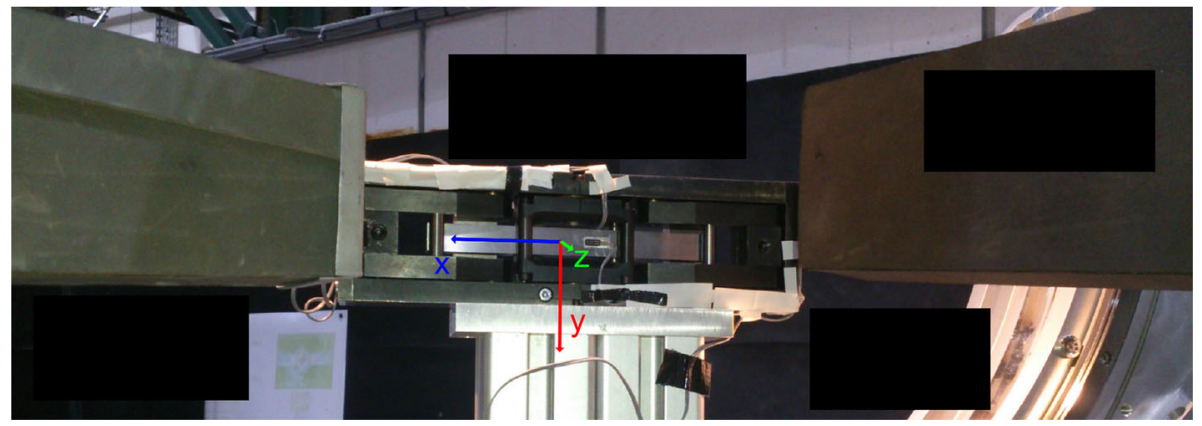

Fig. 4 Experimental four-point-bending setup: The image shows the four-point-bending device mounted on the Eulerian cradle of the SALSA instrument at the neutron research reactor ILL, Grenoble, for the measurements in radial direction of the bending beam. On the left and on the right hand side the radial collimators used on the primary and the secondary neutron beam path, respectively, are partly shown, which finally define the applied nominal gauge volume

the loaded states were referenced to the local lattice parameters determined for the initial (as received) state. Hence, strain differences were determined in relation to the unloaded, initial state. Due to crystallographic texture at some positions and/or some measuring direction unsatisfactory diffraction response occur, which not least contribute to the error bars given for the experimental results. To provide more robust results the $\{\mathrm{hkl}\}$ dependent phase-specific lattice strains were averaged according to the approach proposed in [7], considering the multiplicity factors and the elastic constants calculated on basis of the single crystal parameters given in Table 1. The texture factor was set to 1 . Close to the surface during neutron strain scanning the nominal gauge volume was only partially filled with material, which results in spurious strains. This so-called surface effect was corrected numerically by means of the simulation tool SIMRES [21]. From the phase-specific strains determined this way, stresses were calculated assuming that the axes $x, y$ and $z$ are the principal directions. Here, the texture and the gradient of the texture over the bending height were considered. For stress calculation the effective elastic constants were applied, which were calculated using the phase-specific crystal orientations provided by the ODFs in combination with the single crystal constants $C_{11}, C_{12}$ and $C_{44}$ as given in Table 1 . Since texture analysis was only carried out for a limited numbers of positions over the bending height, the ODF information was interpolated accordingly.

The stress analysis was carried out for loading the bending beam to total strain in the outer layers of $1.54 \%$ and after unloading the samples to monitor the resulting characteristic residual stresses after elasto-plastic four-point-bending.

\subsection{Numerical model}

For the mean field model (MFT) simulation the commercial software Abaqus 2019 from Dassault Systèmes was used. The mean field approach was implemented a user subroutine which is called at each integration point of the beam. The beam is discretized with 25600 three-dimensional incompatible mode continuum elements of linear geometric order (C3D8I), while the rollers are simulated as analytical rigid shells. The symmetries of the beam, boundary conditions and material are utilized, which allows the simulation of a quarter model. The beam is loaded by displacement controlled roller movement with hard and frictionless interaction properties until the effective strain $\bar{\varepsilon}_{x x}=0.02$ is obtained. To illustrate the discretization of the beam Fig. 5 shows the longitudinal component of the effective Cauchy stress $\bar{\sigma}_{x x}$ at stage of maximum load.

With the Taylor ansatz (TJ2), the process is modelled on a two dimensional approximation of an EulerBernoulli beam. Thus an uniaxial stress state at each integration point is assumed. As finite element, the geometrically exact Euler-Bernoulli beam element relies on the kinematics introduced in [5] while certain modifications are made. The element provides a fully nonlinear kinematical framework, while obeying the Bernoulli hypothesis of rigid cross sections and first-order shear rigidity. This results in excellent numerical behavior with respect to modelling thin geometries undergoing large rotations and deformations. Additionally, the element features Rodrigues parametrization of the rotations in an incremental update scheme for numerical robustness and efficiency. Compatibility of Euler-Bernoulli assumptions to a sheet three point bending experiment was shown before as in, e.g., [6]. 

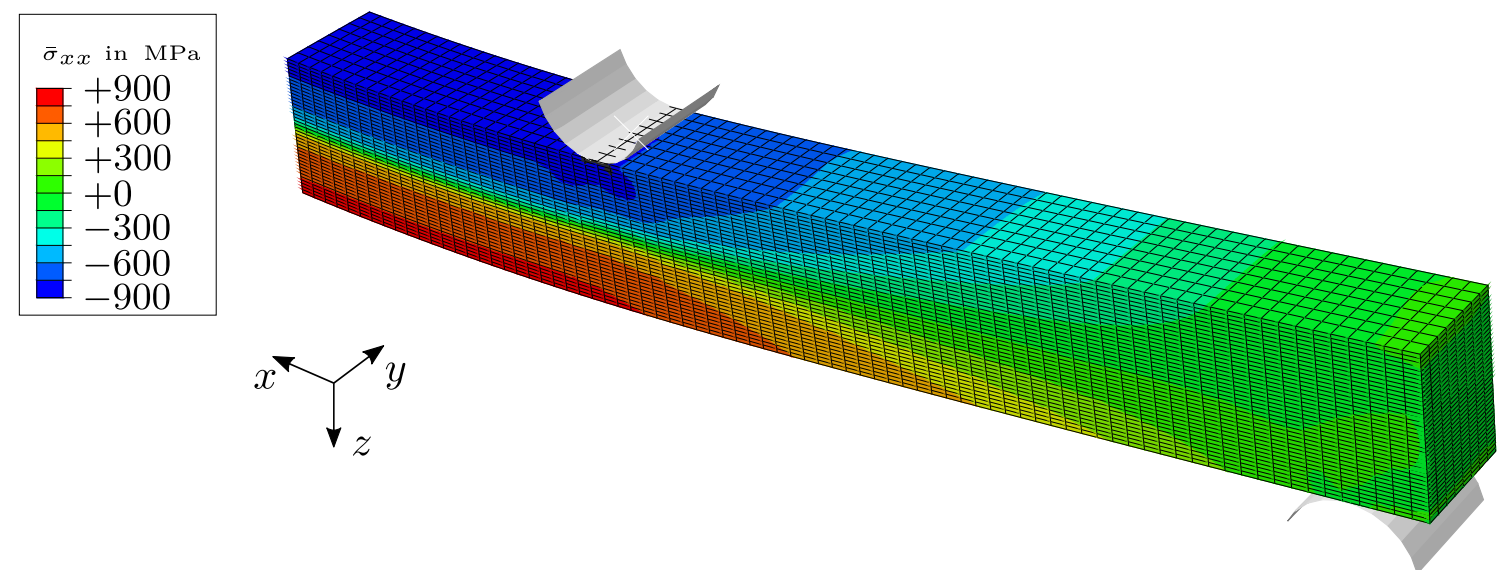

Fig. 5 Longitudinal component of the effective Cauchy stress $\bar{\sigma}_{x x}$ at stage of maximum load based on mean field theory (MFT)

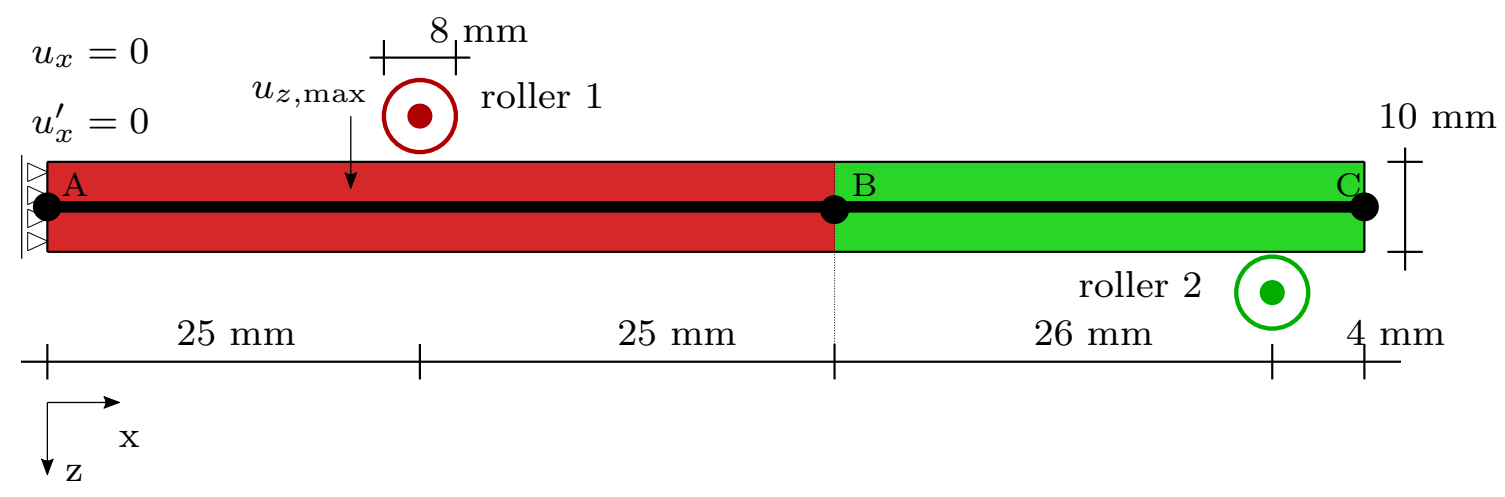

Fig. 6 Boundary value problem for four-point-bending

The element was modified to a point-wise formulation. By performing numerical integration over the cross section and developing a local deformation gradient for each integration point, an interface to arbitrary nonlinear material laws is provided. To ensure the aforementioned zero main lateral stress condition of beams, each integration point provides a local iterative scheme to compute the associated lateral strains. This way the longitudinal strains are obtained directly from the beam kinematics as the main lateral strain is computed within the algorithmic scheme. This procedure was proposed in [12] and successfully applied to shell elements in [25]. The element features 151 Gauss-Lobatto integration points over the cross section height to show the lateral uniaxial stress distribution and to resolves also these quantities at the very top and bottom on the beam.

As model for the contact between the sheet represented by the beam elements and the rollers that introduce the bending a penalty force contact is chosen. The geometry approximation obtained by the beam elements features geometrical $C^{1}$-continuity due to the second Bernoulli hypothesis. In the numerical model we use this property for a very robust algorithm, solving the minimum distance problem. The geometry of the rollers are approximated as rigid disks. Using an implicit representation of the geometrical surface for contact formulations was proposed in [18]. In this contribution we use the concept in conjunction with the $\mathrm{C}^{1}$-continuum geometry of the beam elements but in $2 \mathrm{D}$ only. The resulting consistent contact contributions to the numerical residual and tangent are only projected to displacement degrees of freedom, respectively.

The simulation setup is illustrated in Fig. 6. The beam elements use a geometrical representation for the contact formulation as given in Fig. 6. The contact between the pins and the bending sample was chosen as a hard contact in normal direction and frictionless in transverse direction. Due to the symmetry of the simulation setup only half of the sheet is modelled, using appropriate boundary conditions as depicted in Fig. 6. Additionally, the rollers are illustrated, initially just in contact with the sheet.

The sheet is loaded throughout the simulation by a movement of the rigid roller 1, downwards. After reaching the maximum vertical displacement $u_{z, \max }$ of roller 1 , it is guided back to its original position. Figure 7 shows graphical representations of the beam simulation at critical deformation states throughout the 


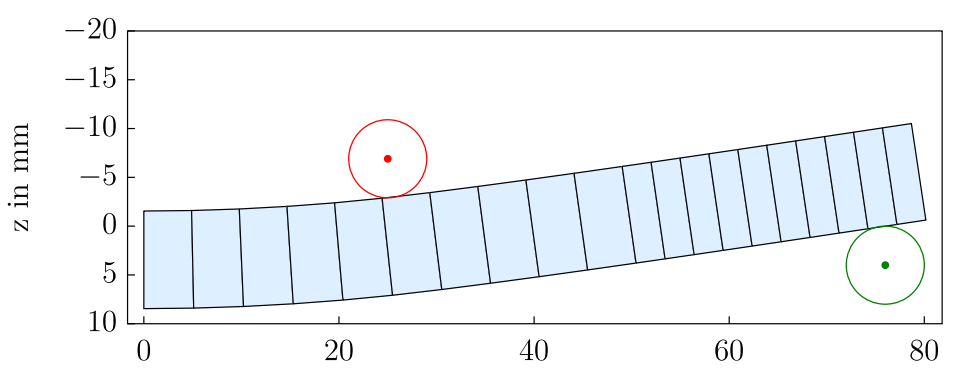

(a)

$\mathrm{x}$ in $\mathrm{mm}$

(b)

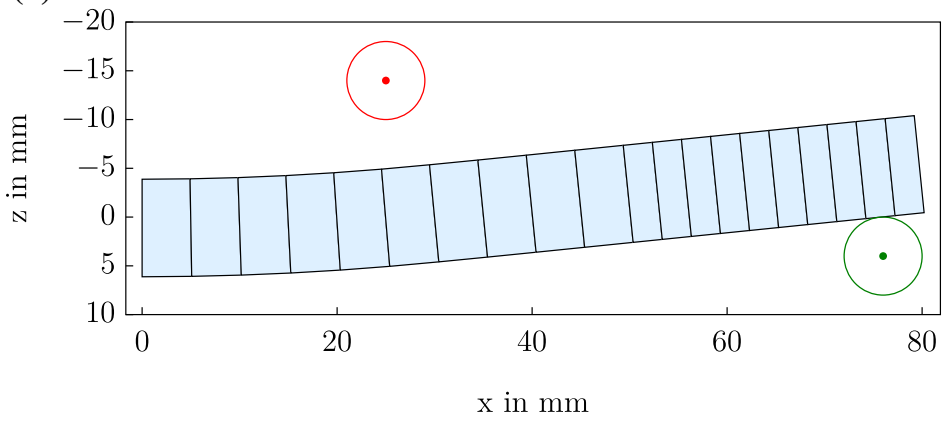

Fig. 7 Bernoulli beam simulation at maximum bending state $\mathbf{a}$ and completely unloaded state $\mathbf{b}$

simulation. The maximum loading $u_{z, \max }$ is chosen to result in a maximum boundary an effective strain of $\bar{\varepsilon}_{x x}=0.02$. This way the unloaded configuration of the sheet and the residual stress distribution after unloading can be investigated.

\subsection{Comparative analysis of in silico and experimental results}

In the following the results of the micro-mechanical finite element simulation are compared to the experimentally obtained results. Experimentally, phase-specific residual stresses were determined by means of neutron diffraction residual stress analysis at ILL@SALSA, Grenoble. For further details on the neutron strain scanning and the experimental setup, it is referred to Sect. 4.1 and to [19]. The stress-strain-distribution for the principal directions will be analyzed over the cross section height of the bending beam. Hereby, we chose the cross section in the geometric center. For both numerical models, this is the cross section at the symmetric support.

In Fig. 8a the longitudinal elastic strain component $\varepsilon_{x x}^{\mathrm{e}}$ over the height of the bending beam $z$ is shown for the austenite $(\gamma-\mathrm{Fe})$ and ferrite $(\alpha-\mathrm{Fe})$ phase at maximal loading. The elastic strain is chosen for the comparison due to the least amount of assumptions in the experimental analysis. Generally, good agreement between all data sources can be observed. The main difference in the numerical results is that the phase-specific longitudinal strains of the mean field model (MFT) are slightly higher than for its counterpart, computed from the Taylor approach (TJ2). The experimental data indicate a shift of the zero crossing in these strains, individually for each of the phases. None of the numerical models show this effect.

More visibly, the experimental data show non-symmetry of the longitudinal strains over the cross section height. The austenite phase shows significantly more compressing strains near the upper beam surface, than tensile strains near the bottom. This effect is less significant for the ferrite phase in the same loading state. Figure $8 \mathrm{~b}$ shows the corresponding elastic strain distribution for the unloaded state. Considering the experimental data as a reference point, the non-symmetry observed in the austenite strains in the loaded case has now expanded. In the compression zone the measurements near the surface are not taken into account, due to aforementioned technical restrictions (compare Sect. 4.1). Nevertheless, it is a clear observation that the occurring residual strains exceed higher amplitude in the lower half of the beam, i.e., in the positive $z$-regime. Both numerical models give very close results, continuing the same trend as in the loading case. While qualitatively the numerical results align with the experimental data, a deviation in magnitude can be observed in 


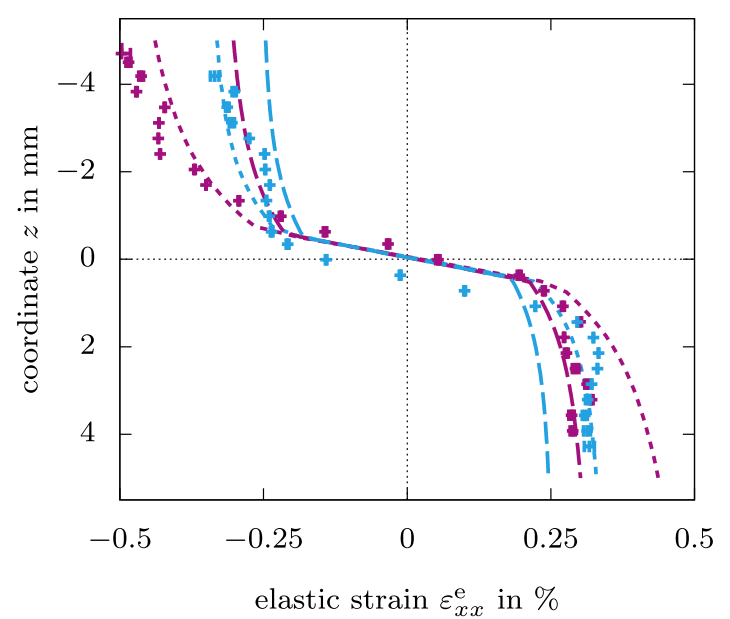

(a) $\varepsilon_{x x}^{\mathrm{e}}$ loaded beam

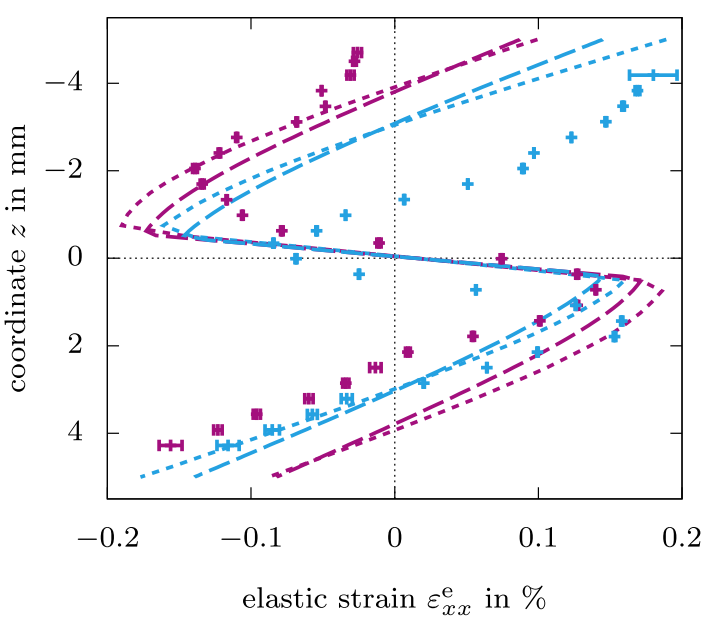

(b) $\varepsilon_{x x}^{\mathrm{e}}$ unloaded beam

$$
\begin{array}{lll}
\gamma \text {-Fe MFT - . . . } & \gamma \text {-Fe TJ2 - } & \gamma \text {-Fe Exp. } \\
\alpha \text {-Fe MFT - - . - } & \alpha \text {-Fe TJ2 }---- & \alpha \text {-Fe Exp. } \longmapsto
\end{array}
$$

Fig. 8 Elastic strains $\varepsilon_{x x}^{\mathrm{e}}$, for the loaded and unloaded state. The results of the finite element simulation (depicted as lines) are shown in comparison to the experimental data obtained by means of neutron diffraction analysis (depicted as points including standard error)

particular for the ferrite phase. This is especially the case as the experimentally measured non-symmetry of the residual strains is not captured by the numerical models.

Before reviewing the residual stresses obtained by the considered models, the lateral strain components are reviewed, see Fig. 9. The error bars that can be assigned to the determined lattice strains and as a consequence also to the calculated stresses are based on statistics that differ with position. The worsening in diffraction statistics leads to an enlargement of the error bars and can be explained by the texture and its distribution over the cross sectional area. Depending on local texture the measured diffraction intensity can be strongly decreased. For some $\{\mathrm{hkl}\}$ diffraction lines it became almost impossible to determine diffraction peaks that are sufficient for a meaningful calculation of the peak position, which is needed for lattice strain calculation. For stress calculation this applies accordingly. This effect of decreased statistics and hence of enlarged error bars is largest at the outer layers, where by means of the texture analysis the sharpest texture exist in particular for the ferrite phase.

Generally, the trend between simulation data and experimental measures continues, as such that overall satisfying agreement can be concluded. Nevertheless, it can be observed that the lateral strains $\varepsilon_{y y}^{e}$ for the unloaded case shows the highest shift with respect to the geometrical center of the beam height. It appears that this is one point, both numerical approaches are not able to account for this phenomenon. The lateral strain distributions on the other hand show a crucial difference in the numerical models. This difference can directly be observed as the thicker depth direction of the beam shows stiffer behavior in that direction $(y)$, as expected. For the lateral $z$ direction, the opposite is the case. The beam element enforces a uniaxial stress condition in each integration point, which benefits numerical performance. Contrary, the solid simulation resolves the Poisson effects directly, which technically is more accurate. Both numerical schemes are also affected by their discretization, i.e., number and positions of integration points. When comparing the numerically approximated strain responses to the measured data, sufficient coverage is observed. When accounting for the actual magnitude of these components compared to $\varepsilon_{x x}^{e}$, it is also clear that their influence on the macroscopic deformations and hence residual stress state is minor. For this reason, we will only focus on the longitudinal stress components for the analysis of residual eigenstresses. However, all discussed data sources use a three dimensional constitutive model, including the residual stress computation from the experimentally measured strains.

Finally, in Fig. 10 the longitudinal stress component over the height of the beam $\sigma_{x x}$ is shown, for the loaded and unloaded state, respectively. 


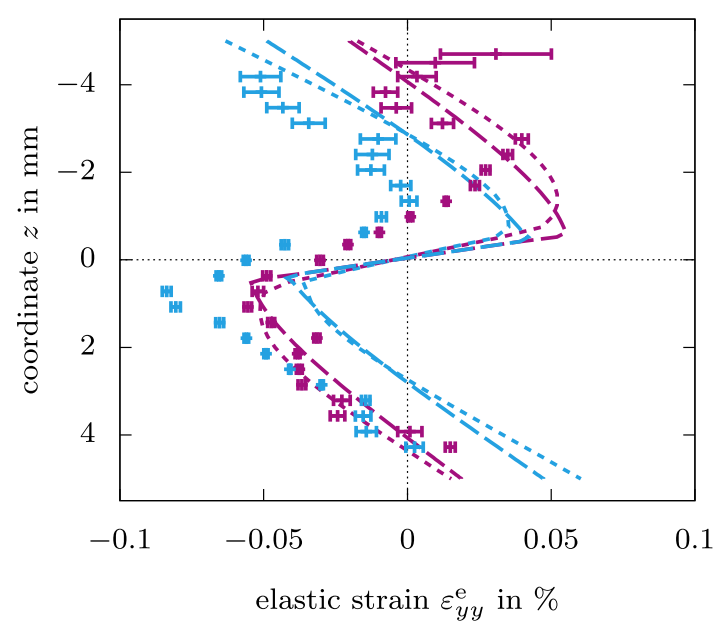

(a) $\varepsilon_{y y}^{\mathrm{e}}$ unloaded beam

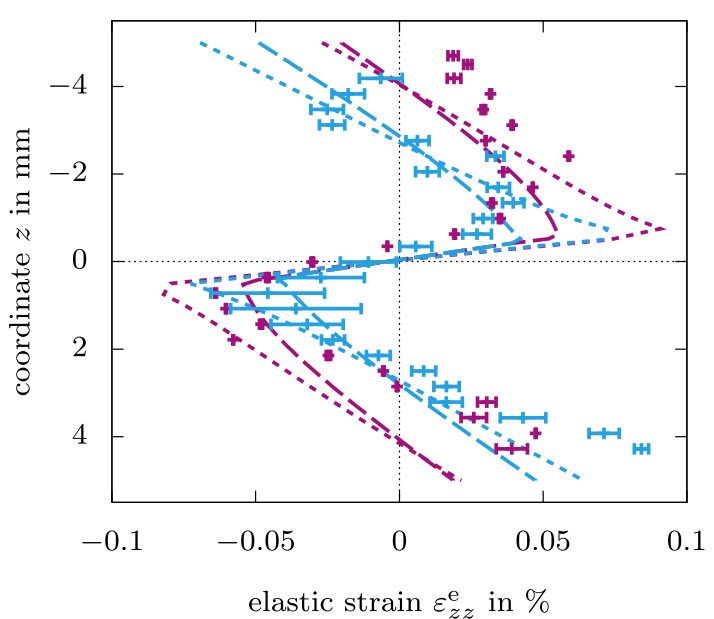

(b) $\varepsilon_{z z}^{\mathrm{e}}$ unloaded beam

$$
\begin{array}{lll}
\gamma \text {-Fe MFT - . . ... } & \gamma \text {-Fe TJ2 - - } & \gamma \text {-Fe Exp. } \\
\alpha \text {-Fe MFT - . - . } & \alpha \text {-Fe TJ2 }---- & \alpha \text {-Fe Exp. }
\end{array}
$$

Fig. 9 Residual elastic strains $\varepsilon_{y y}^{\mathrm{e}}$ and $\varepsilon_{z z}^{\mathrm{e}}$. The results of the finite element simulation (depicted as lines) are shown in comparison to the experimental data obtained by means of neutron diffraction analysis (depicted as points including standard error)

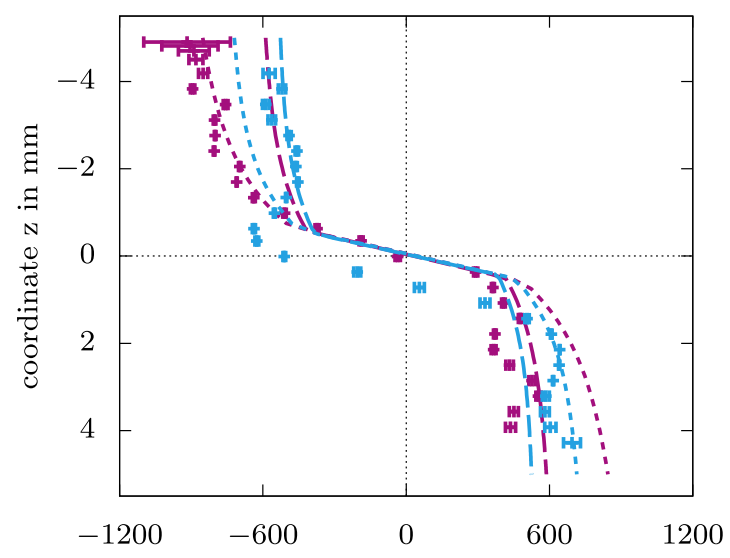

Cauchy stress $\sigma_{x x}$ in $\mathrm{MPa}$

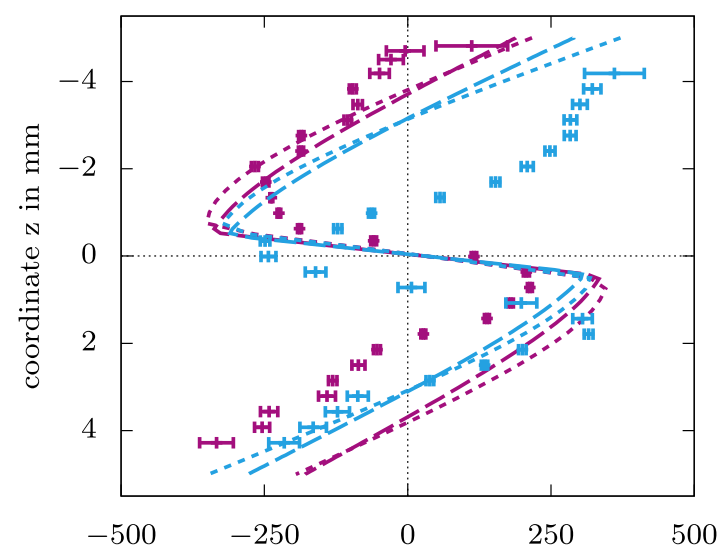

Cauchy stress $\sigma_{x x}$ in $\mathrm{MPa}$

(b) Unloaded beam

(a) Loaded beam

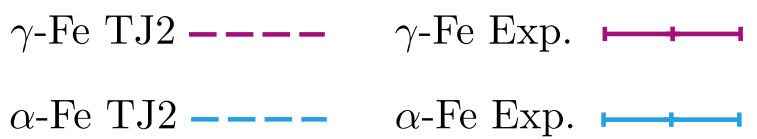

Fig. 10 Stress $\sigma_{x x}$ of the loaded beam (left) and unloaded beam (right). The results of the finite element simulation (depicted as lines) are shown in comparison to the experimental data obtained by means of neutron diffraction analysis (depicted as points including standard error) 
The stress $\sigma_{x x}$ distribution shows similar behavior as observed for the longitudinal strains $\varepsilon_{x x}^{\mathrm{e}}$. This underlines the minor influence of the lateral strain components, which were less well approximated by both numerical schemes. Consequently, good agreement between the experimental data an both numerical approaches can be concluded. Also the magnitudes of stresses are in close proximity, despite the different constitutive models. Both investigated loading states however also show the same weaknesses of the numerical schemes as already observed on the longitudinal strains. The lack of resolving a phase-specific non-symmetry, also in the stresses lead to deviations between numerical stresses, and those determined from experimental data.

\section{Discussion}

In the previous sections we went for a detailed look on the distribution of elasto-plastic stresses and elastic strains for the centre cross section of a beam subjected to four-point-bending. From a macroscopic point of view the simulations following the mean field approach (MFT) and the Taylor ansatz (TJ2) agree very well with the experimentally observed strains and stresses. Nevertheless, some deviations were observed and emphasized in the previous chapter. These were phase-specific shifts of the zero crossing in stresses as well as crucial non-symmetry in these properties. Here, both simulation approaches were not able to predict these, leading to diverging results in some regions compared to the experiment.

The differences can be explained by various effects. Large deviations from smooth stress gradients over the bending height might stem from simplified assumptions in the stress evaluation. Due to the manufacturing process of the rod, from which the bending sample is cut, an inhomogeneous local crystallographic texture occurred as clearly shown by means of cuts through the experimentally determined phase-specific ODFs. However, this local crystallographic texture is not considered in the experimental analysis, nor in the micromechanical framework.

An additional effect might stem from the material parameters used for the micro-mechanical model, listed in Table 1. For the simulation of the forming behavior of coarse two-phase sheet metal materials as close to reality as possible, the determination of the phase-specific material parameters is decisive. In this work the phase-specific material parameters were chosen based on tensile tests combined with X-ray stress measurement. This is a well-justified and meaningful approach. However, since for data evaluation also specific assumptions were made for future work, complementary nano-indentation tests will be performed on the investigated material to support the diffraction data. This attempt failed in the first approach due to the relative small grain size of the two phases and the lower load limitations of the instrumented micro-indentation system at hand. Finally, this allows the determination of more accurate phase-specific hardening behavior and, consequently, a more precise simulation.

Furthermore, the effects of kinematic hardening are not considered in the present work. In the future, experiments under cyclic loading conditions will provide the foundation for determining the necessary material parameters which will be used in the numerical models to account for these phenomena.

The biggest impact is suspected to be found in neglecting the texture of the here investigated duplex steel. When considering Fig. 10b the ferrite phase shows a significant non-symmetric residual stress distribution. A comparison with the ODF scans (see Fig. 3) gives context to this, as especially the ferrite phase exposes a strong non-symmetric texture over the beam height $z$. This inhomogeneous phase-specific texture distribution determined experimentally by means of diffraction analysis, might be the reason for the obvious shift of the neutral fibre in case of the ferrite phase. At the moment we are unfortunately not able to take this, the texture and its inhomogeneous distribution into account during strain and stress evaluation on basis of the diffraction data. This step is part of ongoing work and require the analysis of phase-specific strain pole figures. Regarding the numerical simulation, for higher accuracy in the prediction of residual stresses, a localization scheme must include this phase-specific texture information that was experimentally determined for the real structure. Consideration of the phase-specific local texture in the simulations is essential in order to obtain a realistic numerical prediction of the residual stresses. In Simon et al. [24] an approach is presented, which accounts for an anisotropic material behavior in the mean field homogenization scheme. A correlation of plastic and elastic anisotropies is constructed based on the work of Böhlke et al. [1]. The fourth-order texture coefficient, computed based on the ODF and the crystallographic basis, is assumed to be homogeneous in the specimen. With the ODF sections for different positions of the bending beam, presented in Fig. 3, and the resulting phase-specific residual stress distributions a valuable set of input and validation data is provided for further investigations. 


\section{Conclusion}

A four-point-bending experiment was performed and simulated, considering a beam of the corrosion-resistant duplex steel X2CrNiMoN22-5-3 (1.4462). In aiming to predict the residual stresses in the material after unloading, two different numerical approaches were utilized. For comparison, the simulations were carried out using a numerically efficient mean field approach (MFT) as part of a three dimensional finite element analysis and with a simpler Taylor ansatz (TJ2), on a geometrically exact beam element. Although the mean field theory clearly has more depth and is superior over the Taylor localization, the simulation results are very similar and show good agreement to the experimental data. Nevertheless, some deviations of simulation and experimental data were found and discussed. The investigation clearly shows the influence of texture to the material response. Hence, if more accurate residual stress predictions are required, this texture effect must be included. In this regard the here utilized Taylor ansatz does not offer this capabilities, but the mean field theory will be elaborated to include texture information for future works.

Acknowledgements The authors thank T. Pirling (ILL) for scientific and technical support as well as J. Rebelo Kornmeier (Technical University Munich, Germany) and F. Vollert (Karlsruhe Institute of Technology) for their assistance during the ILL beamtime.

Funding Open Access funding enabled and organized by Projekt DEAL. Funded by the Deutsche Forschungsgemeinschaft (DFG, German Research Foundation) - Project-ID 374768210 (BO 1466/14-2, GI 376/13-2), Project-ID 374871564 (BR 5278/32, SCHR 570/33-2), within the Priority Programme SPP 2013 'Targeted Use of Forming Induced Residual Stresses in Metal Components'. Regarding the experimental part further funding by the DFG was granted through the Project-ID 282874578 (GI 376/11-2). The support by the Deutsche Forschungsgemeinschaft is gratefully acknowledged. Furthermore, the authors are grateful to ILL for granting us beamtime through the project no. 1-02-199.

\section{Declarations}

Conflict of interest On behalf of all authors, the corresponding author states that there is no conflict of interest.

Open Access This article is licensed under a Creative Commons Attribution 4.0 International License, which permits use, sharing, adaptation, distribution and reproduction in any medium or format, as long as you give appropriate credit to the original author(s) and the source, provide a link to the Creative Commons licence, and indicate if changes were made. The images or other third party material in this article are included in the article's Creative Commons licence, unless indicated otherwise in a credit line to the material. If material is not included in the article's Creative Commons licence and your intended use is not permitted by statutory regulation or exceeds the permitted use, you will need to obtain permission directly from the copyright holder. To view a copy of this licence, visit http://creativecommons.org/licenses/by/4.0/.

\section{References}

1. Böhlke, T., Bertram, A., Krempl, E.: Modeling of deformation induced anisotropy in free-end torsion. Int. J. Plast 19(11), 1867-1884 (2003)

2. Bunge, H.J.: Advantages of neutron diffraction in texture analysis. Text. Microstruct. 10, 265-307 (1989)

3. Coleman, B.D., Noll, W.: The thermodynamics of elastic materials with heat conduction and viscosity. Arch. Ration. Mech. Anal. 13(1), 167-178 (1963)

4. Castaneda, P.P.C., Suquet, P.: Nonlinear Composites, pp. 171-302. Elsevier, Amsterdam (1997)

5. da Costa e Silva, C., Maassen, S.F., Pimenta, P.M., Schröder, J.: A simple finite element for the geometrically exact analysis of Bernoulli-Euler rods. Comput. Mech. (2019)

6. Dang, Q.K., Chang, P.L., Kuo, S.K., Wang, D.A.: Prediction of springback and residual stress of a beam/plate subjected to three-point bending. J. Mech. Mater. Struct. 13(4), 421-441 (2018)

7. Daymond, M.R.: The determination of a continuum mechanics equivalent elastic strain from the analysis of multiple diffraction peaks. J. Appl. Phys. 96(8), 4263-4272 (2004)

8. Hill, R.: The elastic behaviour of a crystalline aggregate. Proc. Phys. Soc. Sect. A 65(5), 349 (1952)

9. Hofinger, J., Erdle, H., Böhlke, T.: Prediction of residual stresses of second kind in deep drawing using an incremental two-scale material model. Philos. Mag. 100, 2836-2856 (2020)

10. Kanouté, P., Boso, D.P., Chaboche, J.L., Schrefler, B.A.: Multiscale methods for composites: a review. Arch. Comput. Methods Eng. 16(1), 31-75 (2009)

11. Klages, G.: Zahlenwerte und Funktionen aus Naturwissenschaft und Technik. Neue Serie, Gruppe ÜI: Kristall-und Festkörperphysik. Band 1: Elastische, piezoelektrische, piezooptische und elektrooptische Konstanten von Kristallen. Berichte der Bunsengesellschaft für physikalische Chemie 70(8), 935 (1966)

12. Klinkel, S., Govindjee, S.: Using finite strain 3D-materials models in beam and shell elements. Eng. Comput. 19(3), 254-271 (2002)

13. Ledbetter, H.M.: Predicted single-crystal elastic constants of stainless steel 304. Technical Report, United States. NBSIR81-1645 (1981) 
14. Liu, C.S., Hong, H.K.: Non-oscillation criteria for hypoelastic models under simple shear deformation. J. Elast. 57(3), 201-241 (1999)

15. Macherauch, E., Müller, P.: Das $\sin 2 \psi$-Verfahren der röntgenographischen Spannungsmessung. Zeitschrift für angewandte Physik 13(7), 305-312 (1961)

16. Matthies, S., Humbert, M.: On the principle of a geometric mean of even-rank symmetric tensors for textured polycrystals. J. Appl. Crystallogr. 28(3), 254-266 (1995)

17. Miehe, C., Rosato, D., Frankenreiter, I.: Fast estimates of evolving orientation microstructures in textured bcc polycrystals at finite plastic strains. Acta Mater. 58(15), 4911-4922 (2010)

18. Neto, A.G., Pimenta, P.M., Wriggers, P.: A master-surface to master-surface formulation for beam to beam contact. Part i: frictionless interaction. Comput. Methods Appl. Mech. Eng. 319, 146-174 (2016)

19. Pulvermacher, S., Gibmeier, J., Šroun, J., Rebelo Kornmeier, J., Vollert, F., Pirling, T.: Neutron strain scanning of duplex steel subjected to 4-point-bending with particular regard to the strain free lattice parameter d0. In: Residual Stresses, 11. September 2018. Ed.: M. Seefeldt, Materials Research Proceedings, vol. 6, pp. 15-20. Materials Research Forum LLC, Millersville (PA) (2018)

20. Richard, D., Ferrand, M., Kearley, G.J.: Analysis and visualisation of neutron-scattering data. J. Neutron Res. 4(1-4), 33-39 (1996)

21. Šaroun, J., Kulda, J.: Raytrace of Neutron Optical Systems with RESTRAX, pp. 57-68. Springer, Heidelberg (2008)

22. Schajer, G.S.: Relaxation methods for measuring residual stresses: techniques and opportunities. Exp. Mech. 50(8), 11171127 (2010)

23. Simo, J.C.: Algorithms for static and dynamic multiplicative plasticity that preserve the classical return mapping schemes of the infinitesimal theory. Comput. Methods Appl. Mech. Eng. 99, 61-112 (1992)

24. Simon, N., Krause, M., Heinemann, P., Erdle, H., Böhlke, T., Gibmeier, J.: Phase specific strain hardening and load partitioning of cold rolled duplex stainless steel X2CrNiN23-4. MDPI Crystals (submitted)

25. Viebahn, N., Pimenta, P.M., Schröder, J.: A simple triangular finite element for nonlinear thin shells: statics, dynamics and anisotropy. Comput. Mech. (2016)

26. Willis, J.R.: Variational and Related Methods for the Overall Properties of Composites, pp. 1-78. Elsevier, Amsterdam (1981)

27. Zienkiewicz, O.C., Taylor, R.L.: The Finite Element Method for Solid and Structural Mechanics. Elsevier, Amsterdam (2005)

Publisher's Note Springer Nature remains neutral with regard to jurisdictional claims in published maps and institutional affiliations. 